\title{
Wann ist die Gefahr für eine postoperative Pneumonie besonders hoch?
}

\author{
Ziel des Deutschen Thoraxregisters ist es, die Behandlungsqualität bei Thoraxeingriffen zu \\ verbessern. Im Oktober 2016 wurde das Teilnehmerspektrum erweitert. Jetzt sind sämtliche \\ Kliniken mit mindestens 50 thoraxchirurgischen Eingriffen im Jahr teilnahmeberechtigt. \\ In München wurden erste Ergebnisse vorgetragen.
}

Nach Ausarbeitung eines umfangreichen Datenschutzkonzeptes und Abschluss der Pilotphase wurde das Deutsche Thoraxregister als erstes perioperatives, interdisziplinäres Behandlungsregister im Januar 2016 offiziell eröffnet. Neben der reinen Registerforschung profitieren die teilnehmenden Kliniken vor allem durch die „Benchmarking“-Funktionen, also durch den Vergleich der Behandlungsqualität verschiedener Kliniken.

Wie Dr. Mark Schieren von den Kliniken Köln erläuterte kann anhand der gesammelten Daten eine kritische Prüfung der Sicherheit, Wirksamkeit und Wirtschaftlichkeit klinischer Behandlungskonzepte an großen Untersuchungskollektiven erfolgen. Die Erkenntnisse aus Registerdatenbanken entsprechen dem tatsächlichen Patientenklientel der teilnehmenden Kliniken und sind nicht durch strenge Einschlusskriterien sonstiger klinischer Studien auf kleinere Subgruppen beschränkt: ein entscheidender Beitrag zur Optimierung der Versorgungsqualität in der deutschen Thoraxchirurgie.

\section{Erste Ergebnisse}

Im Register waren Mitte Oktober 2017 die Daten von 809 Patienten gespeichert, davon 59,6\% Männer. Bei 678 Patienten erfolgten Lungenresektionen. Hierbei kam es bei 12,2\% nach der Operation zu einer Pneumonie. Dies entspricht Schieren zufolge einem Fall an jedem zweiten Tag.

Das präoperative Assessment erfasste Basisdaten (Alter, Geschlecht), Lebensstilfaktoren wie Rauchen, die Lungenfunktion $\mathrm{FEV}_{1}$ und Diffusion, Laborwerte wie Blutgase und CRP, auch die OP-Planung: VATS oder Thorakotomie.

Eine erste Auswertung stellte die Frage nach einem erhöhten postoperativen Pneumonierisiko.

Hier die Ergebnisse: Zur postoperativen Pneumonie kam es bei $15,1 \%$ der Männer und 8,5\% der Frauen $(p=0,013)$. Das männliche Geschlecht gilt deshalb als (naturgegebener) Risikofaktor.

Die Staffelung des Risikos für eine postoperative Pneumonie bei Rauchern: Nichtraucher 10,1\%, Exraucher (seit über 3 Monaten) $11,8 \%$, aktive Raucher $13,8 \%$. Das ist statistisch nicht signifikant. Was allerdings nicht missdeutet werden sollte: Rauchen schadet der Lunge per se.

\section{Lungenfunktionswerte}

- $\mathrm{FEV}_{1}$ (Einsekundenkapazität, forciertes expiratorisches Volumen): Patienten mit $>70 \mathrm{FEV}_{1}$ bekamen in $10 \%$ eine $\mathrm{pP},<70$ waren es $18 \%$. Sprich, eine $\mathrm{FEV}_{1}<70$ bedeutet Risikofaktor für eine postoperative Pneumonie.
- Lungendiffusionsdiagnostik, um festzustellen, wie gut die Atemgase von den Alveolen ins Blut übertreten (zur Bestimmung des Schweregrads von Emphysemen): Lag der Diffusionswert bei $<60$, betrug das Risiko 20,2\% - also ebenfalls ein Risikofaktor für eine postoperative Pneumonie.

\section{Laborwerte}

- Das größte Einzelrisiko erreichte ein $\mathrm{pO}_{2}$ von $<60 \mathrm{mmHg}$ mit $37,5 \%$. Aber auch bei $\mathrm{pO}_{2}<70$ lag es noch bei $15,3 \%$.

- Die gemessenen Unterschiede beim arteriellen $\mathrm{pCO}_{2}$ waren statistisch nicht signifikant. Dagegen erreichte ein CRP-Wert von $>3,0 \mathrm{mg} / \mathrm{l}$ die Signifikanzgrenze mit einem Risiko von $15,6 \%$, bei $>40$ erreichte das Risiko sogar $20 \%$.

\section{Operationstechnik hat großen Einfluss}

Besonders spannend war die Frage, unter welcher OP-Technik ein erhöhtes Risiko für eine postoperative Pneumonie droht. Das Ergebnis war eindeutig: Unter der Thorakotomie 22,1\%, bei dem minimalinvasiven Eingriff (VATS) $9 \%(\mathrm{p}=0,027)$.

Wie zu erwarten stieg das Gesamtrisiko für eine postoperative Pneumonie mit der Zahl der Risikofaktoren, und zwar fast linear bis zur Summe von vier Risikofaktoren auf 17,9\%. Werden jedoch fünf Faktoren erreicht, schnellt das Gesamtrisiko auf $38,1 \%$ hoch.

Der Referent vermied es, diese ersten Ergebnisse gleich als Alarmsignale zu verkünden. Vielmehr stehe das gesamte statistische Werk auf schwachen Füßen, es sollten daraus nur Trends abgelesen werden. Um die Ergebnisse zu stabilisieren, bedarf es nach Schieren noch mehr Fälle und mehr Zentren, die sich am Register beteiligen.

Dr. med. Jochen Aumiller

\section{Deutsches \\ Thoraxregister}

Deutsches Thorax-Register

Am Deutschen Thorax-Register können alle Kliniken teilnehmen, die im Jahr mindestens 50 Eingriffe am Brustkorb vornehmen. Inbegriffen sind eine standardisierte Datenerhebung, zeitnahe Analyse sowie ein webbasiertes Feedback. Mehr Informationen zum Register und den Teilnahmebedingungen finden Sie im Web unter www.thoraxregister.de 